\title{
Beitrag zur Kenntniss der alpinen 0rthopterenfauna.
}

\section{Von Dr. Hermann Krauss in Tübingen.}

Bei einem Anfangs September dieses Jahres ausgeführten Besuch des südöstlich von Hindelang im bayrischen Algäu gelegenen, von der Osterach durchflossenen, durch 5-7000 Fuss hohe Berge fast gänzlich von der Aussenwelt abgeschlossenen Thales von Hinterstein (hinter dem Stein!) lernte ich an den Ufern des genannten Gebirgsbaches eine merkwürdige, entschieden "autochthone" Orthopterenfauna kennen, deren ausführlichere Besprechung namentlich mit Rücksicht auf die geographische Verbreitung der einzelnen Arten mir von Interesse erscheint.

Der Haupt-Fundplatz liegt in unmittelbarer Nähe des an der östlichen Thalseite lang hingestreckten Dörfchens Hinterstein (2650 F. ü. M.) und wird durch das aus Geröllfeldern bestehende rechte Ufer der Osterach, die hier zugleich mit denselben die Thalsohle fast gänzlicb ausfüllt, gebildet. Während das Geröll gegen das Wasser zu des Pflanzenwuchses vollständig entbehrt, trägt es weiter zurüick eine spärliche, vorzugsweise alpineVegetation. Zwischen niedrigem Weidengebüsch und einzelnen Tannenbäumchen finden sich auf Geröll oder Sand da und dort Rasen von Dryas octopetala, Saxifraga aizoides, Linaria alpina, Tussilago alpina, Hieracium staticefolium etc. Und gerade hier aber auch auf den vollständig vegetationslosen Geröllfeldern treiben sich zahlreiche Orthopteren in bunter Abwechslung herum, während sie auf den benachbarten üppigen Wiesen fehlen.

Hier traf ich zuerst auf spärlich bewachsenem Geröll oder Sand den äusserst behenden, im Leben hübsch gefärbten, seltenen Stenobothrus pullus Philippi in Menge, dazwischen hüpfte da und dort der unscheinbare Tettix Türki Krauss, eine Art, die bisher in Deutschland nicht beobachtet worden ist. Die Königin des Tags war aber die grösste und schönste, aber auch seltenste europäische Oedipodide Bryodema (Oedipoda) tuberculata Fab., deren Männchen beim Fliegen schon von weitem ihr merkwürdiges, fast metallisch klingendes Schnattern hören liessen. Sie flogen überall auf vom sterilen Geröll, wie von den mehr bewachsenen Orten und erhoben sich zuweilen in Spiralen aufsteigend unter fortwährendem Schnattern hoch in die Luft. Auch die plumperen Weibchen 
liessen sich $a b$ und zu zum Fliegen an, wobei sie gleichfalls einen schnatternden, aber schwächeren Ton von sich gaben, ohne jedoch den überaus fluggewandten Männchen gleich zu kommen. Dazwischen flogen oder hüpften neben zahlreichen Cicindelen Stenobothrus bicolor Charp., Psophus (Gryllus) stridulus L. und einzelne Platycleis brachyptera L. in der überaus seltenen Varietät mit vollständig ausgebildeten Flugorganen. Es war ein buntes Bild voll Leben, das jeh auf sterilem Boden im stillen Gebirgsthal nicht erwartet hatte.

Die beiden erstgenannten Arten (Sten. pullus und Tett. Türki) sind für das bayrische Gebirge neu, letztere war bisher überhaupt nicht als alpine Art bekannt und ist, wie schon gesagt, für Deutschland neu, Bryodema tuberculata dagegen wurde ohne $\mathrm{Z}$ weifel zuerst im Jahre 1869 von $\mathrm{E}$. $\mathrm{H}$ o fma n $\mathrm{n}$ (Stuttgart) im Hintersteiner Thal aufgefunden, wie ich dies in Verh.Zool. Bot. Ges. Wien, XXIII, 1873, pag. 24, und ebenda XXXIII, 1883, pag. 220, erwähnt habe, nur dass statt Hinterstein das bekanntere nahe gelegene Hindelang als Fundort angegeben wurde. Sie mögen insbesondere bezüglich ihrer Verbreitung ausführlicher besprochen werden.

\section{Stenobothrus pullus Phil.}

Diese von Philippi auf der Jungfernhaide bei Berlin entdeckte und als Gryllus pullus in Orthoptera Berolinensia 1838 beschriebene und abgebildete Art, die nach Brunner nur „im nördlichen und östlichen Europa" vorkommt, gehört, wie der neueste Fundort beweist und wie auch der von mir bei Wattens am rechten Innufer unterhalb Innsbruck entdeckte Fundplatz (Verh. Zool. Bot. Ges. Wien, XXIII, 1873, pag. 23), sowie das von Brunner angegebene Vorkommen in Kärnthen vermuthen liessen, entschieden auch der alpinen Fauna an und ist unter jene Arten $\mathrm{zu}$ stellen, die im nördlichen und östlichen Europa in der Ebene, im Süden dagegen im Gebirge vorkommen, während sie im mittleren Europa fehlen oder nur vereinzelt $\mathrm{zu}$ finden sind.

Die bisher bekannten Fundorte sind:

Norddeutschland: Berlin, Jungfernhaide (Phil.), Harz (Rudow);

Mitteldeutschland: Sachsen (Fieber), Sächsische Schweiz, Dresdener Haide, Hohenwiese, Leuben (W a n ckel);

Süddeutschland: Regensburg (Herrich-Schäffer). 
Oesterreich: Oberschlesien (Kelch), Böhmen (Fieber), Wien, Kärnthen (Brunner), Tirol, Innthal bei Wattens (Innsbruck) (Krauss), Galizien (Brunner), Siebenbürgen (Herman).

Russland: Kasan (Eversmann), Tiflis (Steven, Fisch. W.).

Sie findet sich ausgewachsen von Ende Juli bis October immer nur auf sterilen sonnigen Plätzen, Haiden, sandigen Flussufern, trockenen Hügeln und Feldern, im Kasan'schen nach Eversmann „auf sandigen, grasbewachsenen freien Stellen in Tannenwäldern " und ist äusserst behend im Springen, während sie von ihren kurzen Flügeln keinen Gebrauch macht. Die Varietät mit ausgebildeten Flugorganen scheint selten, sie wurde von mir nicht beobachtet, dagegen von Brunner erwähnt. W a nckel fand sie (q) in der sächsischen Schweiz.

2. Bryodema (Oedipoda) tuberculata F a b.

Fabricius beschrieb diese Art aus Jütland schon im Jahre 1775 in Syst. Entomologiae, pag. 290, und nicht wie bisher angenommen wurde (Brunner, Fischer) erst in Spec. Iv sect. 1781 oder in Entomologia system. II, 1793. Aber offenbar ist $\mathrm{Pallas}$ ihr eigentlicher Entdecker, der sie schon in den Jahren 1769-1772 in den Steppenländern des südlichen Russlandes und Süd-Sibiriens auffand, sie jedoch nicht als neue Art erkannte, sondern fälschlicherweise als Gryllus obscurus L., ein Name, mit welchem Linné eine südafrikanische, jetzt in's Genus Pycnodictya gestellte Oedipodide bezeichnete, aufführt. (Pallas, Reise durch verschiedene Provinzen des russ. Reichs. St. Petersb. 1.-3. Theil, 1771-1776.) Obgleich er keine Beschreibung gibt, so geht aus seiner Schilderung des Fluges und des dabei hervorgebrachten Geräusches mit Sicherheit hervor, dass er unsere Art vor sich hatte. Bestätigt wird dies auch durch Stoll, der ein von Pallas aus Sibirien zurückgebrachtes $O$ beschreibt und abbildet (Stoll, Representation etc. 1787-1813. Sauterelles p. 22, Pl. IX, b. Fig. 32), freilich unter dem Namen Gryllus dilutus und ohne des Pallasschen Namens zu erwähnen. Dasselbe ist übrigens auch bei dem nächstverwandten, von Pallas zuerst beschriebenen Gryllus (Bryodema) barabensis der Fall, den Stoll gleichfalls nach einem von Pallas gesammelten Exemplar unter dem neuen Namen Gryllus luctuosus beschreibt und abbildet, woraus hervorgeht, dass er die von jenem gebrauchten Namen eben einfach nicht kannte. - Auch in: „Bemerkungen auf 
einer Reise in die südl. Statthalterschaften des russ. Reichs in den Jahren 1793 und 1794", Leipzig, 1. Bd., 1799, p. 337, erwähnt Pallas noch einmal unsere Art vom Nordabhang des Kaukasus, wo er sie bei der Festung Constantinogorsk (Pjatigorsk) am Bache Kosada (11. Sept. 1793) fand: "Ich sah hier wieder die in Sibirien gemeinen Gryllos obscurus, fuscus, coerulescens und stridulus häufig, aber grösser wie gewöhnlich."

Abgesehen von ihrer Grösse, Färbung und ihrem merkwürdigen Fluge ist die Art durch ihre Verbreitung höchst interessant, indem sie sich in einem grossen Theil der paläarctischen Region, und zwar in 3 Subregionen derselben zw ischen dem $60.0^{\circ}$ und $40 .{ }^{\circ} \mathrm{n}$. Breite vorfindet.

I. In der europäischen Subregion (Nord- und CentralEuropa) wurde sie in 5 verschiedenen, zum Theil weit auseinander gelegenen Gegenden gefunden:

1. in Dünemark: Jütland "habitat in Jutlandiae arenosis" (F a b.), Seeland (Brunner);

2. in Norddeutschland: Lüneburger Haide (Heyer), Hamburg (Brunner), Mecklenburg an der Peene (Rudow), Berlin (B urmeister), Frankfurta. O. (Philippi), Glogau (Zeller); 3. in Fialand: Finland (de B orck), Ladoga-See (H i s inger), \lömmö (S a hlberg);

4. am Nordabhang der Alpen: Bayrische Alpen, Hinterstein bei Hindelang ( $\mathrm{H}$ o f mann), Isar- und Riss-Thal, 3-4000 F. ü. M. (Graber), Nordtirol, Plan-See (Leydig), Reutte (Krauss), Tirol, 5-6000 F. ü. M. (Ros enhauer);

5. im südöstlichen Russland: Pallas fand sio im Juni 1769 auf den Bergen bei Samara und in den Steppen gegen Orenburg zu, sowie im Juli desselben Jahres auf den Guberlinski'schen Bergen und bei Orsk, im August 1770 auf den Steppen bei Troizk (Orenburg), Eversmann bei Kasan, Simbirsk, Samara, Saratow, Orenburg und in den Vorgebirgen des Ural, also zum Theil an denselben Orten wie jener, ausserdem um Sarepta. KirgisenSteppe, Bogdo-Berg (Kittary), Caspisches Meer (Brunner), Kaukasus (Pallas, Motschulsky).

II. In der sibirischen Subregion sind folgende Fundorte bekannt: Staniz Kriwoserskoi zwischen Omsk und Semipalatinsk, Argun an der mongolischen Grenze (Pallas), Barnaul (Finsch, nach einem von ihm zur Ansicht erhaltenen Exemplar!), Irkutschk (Sedak off), Transbaikalien, Amur (Brunner). 
III. Aus der manschurischen Subregion erhielt sie Brunner von Nordchina, das kaiserliche Museum in Wien aus Peking (Ransonnet).

Ihre eigenthümliche Verbreitung in der europäischen Subregion weist darauf hin, dass wir es hier mit einem Glied der nautochthonen" Fauna zu thun haben, das in Europa durch Cultur und andere Einflüsse in seinem Vorkommen wesentlich eingeschränkt worden ist, dagegen in den südrussischen und sibirischen Steppenländern noch jetzt seine ungestörte weitausgedehnte Hauptheimat hat.

Hier findet sie sich hauptsächlich in der eigentlichen Steppe, und zwar von der Niederung bis auf die Berge, im Kasan'schen nach Eversmann auch auf sandigen Stellen in lichten Tannenwäldern. Im nördlichen Europa ist es das Haideland, das ihre Heimat bildet, während ihr in den Alpen die Flussthäler mit ihren breiten von Geröll und Sand bedeckten Betten Steppe und Haide ersetzen.

$\mathrm{P}$ allas ist, wie oben gesagt, der Erste, der sie im Freien beobachtete und über ihren "rauschenden" oder "schnatternden" Flug berichtet. Er thut dies mit folgenden Worten gelegentlich der Schilderung seiner Reise über die Guberlinskischen Berge: "Bis einige Werste vor der Redut Rasboinoi fährt man noch immer bergan, über lauter felsige dürre Höhen, auf welchen ansehnlich grosse Heuschrecken (Gryllus obscurus) mit rothen Flügeln, womit sie im Fluge stark zu rauschen pflegen, zerstreut herumzogen" (Reise durch versch. Prov. etc. 1. Theil, 1771, pag. 258); ferner in der Beschreibung seiner Reise entlang der mongolischen Grenze: „Im Junius verdorren die Berge und hohen Steppen um den Argun fast ganz und schwärmen voll Heuschrecken (Gryllus obscurus und barabensis), welche die Wetterveränderung durch ihren schnatternden Flug hoch in der Luft richtig anzeigen" (ibid. 3. Theil, 1776, pag. 433).

Nach Eversmann (Bullet. Soc. Natural. Moscou, T. 32, P. 1, 1859, pag. 140), der sie in Südrussland beobachtete, kreisen einzelne „hoch in der Luft, mit starkem schnatternden Geräusch lange umher, besonders gegen Abend, so dass der Unkundige nicht begreift, woher der Lärm". Auch Leydig (Verh. d. natur. Vereins d. preuss. Rheinl. u. Westph. XXXVIII. Jahrg. (4), VIII. Bd., 1881, Separat., pag. 139) be- 
obachtete sie am Plansee in Nordtirol genauer, wobei ihm "das Thier zunächst dadurch auffiel, dass es bei Südwind und bevorstehendem Regenwetter hoch in der Luft unter starkem Geschnarre lang umherflog, ehe es sich einmal niederliess."

Den ausführlichsten Bericht liefert Graber (Sitz.-Ber. k. Akad. Wissensch., Wien 1872. Separat., pag. 7-9, Fig. 2), der sie im September ungemein häufig in Gesellschaft von Psophus stridulus auf dem breiten mit Kalkgeröll und zum Theile mit Zwergkiefern bedeckten Bett der Riss und Isar in einer Seehöhe von ungefähr 3-4000 Fuss beobachtete:

"Schon in aller Frühe (zwischen 6 und $7 \mathrm{Uhr}$ ) flog unsere Oedipoda oft ununterbrochen gegen eine Viertelstunde lang so hoch in den Lüften herum, dass ich sie nicht selten fast ganz aus dem Auge verlor. Dabei hebt und senkt sie ihre Flügel ganz rhythmisch wie ein echter Vogel und sehwebt so in lang gezogenen Wellenlinien langsam auf und nieder. Gleichzeitig mit diesen regelmässigen Flugbewegungen, welche ich bisher bei keiner einheimischen Form beobachten konnte, lässt unser Acridier ein ganz harmonisch klingendes, sehr lang gedehntes schrrr, schrrr hören. Auch das Weibchen macht Musik, wenigstens habe ich solches beim Auffliegen vernommen. Der diesbezügliche Ton lässt sich am ehesten mit dem Geklapper von Pachytylus stridulus vergleichen, klingt aber viel weicher."

Graber verbreitet sich sodann über die Construction der Unterflügel, die sie zum anhaltenden Fluge wie zu den dabei hervorgebrachten lauten Tönen befähigen. "Dieselben bilden umfangreiche Fächer", deren Radialvenen alternirend bald schwächer, bald sehr kräftig entwickelt sind. Ob das Klappern beim Fluge durch die Reibung der vordersten Flügelrippen an der Unterseite der Oberflügel zu Stande komme oder aber durch das gewaltsame Zusammenschlagen der fächerartigen Unterflügel, lässt er unentschieden.

Dieser ausführlichen Schilderung habe ich wenig beizufügen. Die Zeit meiner Beobachtung über Mittag war zu kurz und der betreffende Tag insofern ungünstig, als eine drückende Hitze, auf die ein schweres Gewitter folgte, die Thiere offenbar beeinflusste. Sie zeigten sich zwar äusserst lebhaft, flogen schnatternd da und dorthin, nur wenige Männchen aber erhoben sich, weite Spiralen beschreibend, hoch in die Luft, kehrten aber sofort wieder auf den Boden zurück. Die Weibchen er- 
hoben sich fliegend nur wenig über denselben und liessen sich nach kurzem Fluge wieder nieder. Ununterbrochen ertönte während des Fliegens bei beiden Geschlechtern das eigenthümliche, am ehesten mit Schnattern zu bezeichnende Geräusch, das bei den Männchen viel stärker und etwas metallisch klingend erscheint, während es bei den Weibchen bedeutend schwächer und ohne Klang ist.

Trotz ihres gewandten weiten Fluges ist ihr Fang mit dem Netze nicht allzu schwierig und insbesondere die plumperen Weibchen sind leicht zu erhaschen, da sie häufig gar nicht auffliegen, sondern hüpferd zu entfliehen suchen. Sehr schwierig ist es aber, sie, sobald sie ganz ruhig am Boden sitzen, was häufig geschieht, von demselben zu unterscheiden, da ihre graubraune fleckige Färbung ganz auffallend mit der des Gerölls und Sandes übereinstimmt. Die schützende Aehnlichkeit ist hier nicht $\mathrm{zu}$ verkennen und sie wissen sie durch ruhiges Sitzenbleiben auszunützen.

Ueber ihre Nahrung ist mir nichts bekannt geworden und auch kein anderer Beobachter erwähnt sie. Köppen (Die schädlichen Insecten Russlands. St. Petersburg 1880, pag. 97) ist der Einzige, der sie als in Norddeutschland schädlich auftretend bezeichnet, ob mit Recht, ist mir zweifelhaft, da ich sonst hierüber in der Literatur nichts erwähnt finde.

\section{Tettix Türki $\mathrm{Kr}$ auss.}

Von der Farbe des Sandbodens, auf dem sie lebt, ist diese Art sehr leicht zu übersehen und dürfte es daraus zu erklären sein, dass sie bisher nur an 3 weit von einander entfernten Orten aufgefunden wurde.

Sie wurde von R. Türk bei Wien an flachen, sandigen, spärlich bewachsenen Uferstellen der Donau entdeckt und von mir nach an den Altwassern der Donau bei Floridsdorf selbst gesammelten Exemplaren im Jahre 1876 in den Entomolog. Monatsbl. (Kraatz) I, Nr. 7, pag. 103 beschrieben. Später fand sie Brunner an den Ufern der Morava und Moravitza in Serbien und Cobelli an der Etsch bei Roveredo in Südtirol. Auch von den beiden letzteren Fundorten liegen mir Exemplare vor. Ihr Vorkommen bei Hinterstein ist insofern sehr interessant, als es den Beweis liefert, dass ihre eigentliche Heimat die Alpenthäler sind und dass sie von da entlang der Flüsse in die Ebene herabsteigt. 
Sie ist ein echtes Uferthier, das nur auf Sand und Geröll sein Dasein fristet. Durch ihre Färbung und grosse Behendigkeit entgeht sie Nachforschungen leicht. Sie findet sich nach den Beobachtungen Türk's und Cobelli's von April bis September ausgewachsen und soll nach ersterem im letzten Stadium der Verwandlung überwintern, um dann im Frühjahr als vollkommenes Insect zu erscheinen. Ich fand am 3. September bei Hinterstein neben zahlreichen fertigen $\mathrm{T}$ hieren, die sämmtlich zu der Form mit kurzem Pronotum-Fortsatz gehören, einzelne äusserst kleine Larven der beiden ersten Stadien.

\section{Beitrag zur Fauna der zweiflügeligen Insecten.}

Von Dr. H. Dziedzicki in Warschau.

(5. Fortsetzung.)

\section{Mycetophila guttata n. sp. (pag. 12.)}

Synon. : Mycet. signata Winn. pt. Bei dieser Art, von welcher ich 10 Stïcke besitze, sind öfters als bei anderen verwandten Arten die 3 Rückenstriemen untereinander in der Gestalt eines W vereinigt; es gibt aber auch Stücke, bei welchen diese Striemen ganz zusammengeflossen erscheinen. Die Flügelbinde endet oft nicht in der 1 . Hinterrandzelle, sondern geht als senkrechter Schatten zum hinteren Aste der Discoidalgakel herab. Uebrigens ist diese Art dermassen der Mycet. sigillata m. ähnlich, dass die Unterscheidung nur durch den verschiedenen Bau des Hypopygs ermöglicht wird: die lam. bas. ist von unten sehr tief bogenförmig ausgeschnitten; forc. sup. 3-armig, der obere Arm schmal, lang, der untere lang und breit, unten mit 2 grösseren Borsten versehen, der äussere Arm breit und kurz. (Taf. VII, Fig. 25-28.)

\section{Mycetophila signatoides n. sp. (pag. 13.)}

Synon.: Mycet. signata Winn. pt. Sie ist der Mycet. sigillata $\mathrm{m}$. vollkommen ähnlich und unterscheidet sich nu $\mathrm{r}$ durch den Bau des Hy po pyg s: forc. sup. 3-armig, am Innenrande mit 4 Borsten; forc. inf. von oben tief ausgeschnitten, so dass 2 längere Fortsätze entstehen, deren äusserer breiterer an der Spitze einen quadratförmigen, breiten, kurzen, völlig stumpfen Dorn trägt; unten ist die Zange mit 2 Borsten versehen. (Taf. VIII, Fig. 9, 10.)

9. Mycetophila mohilevensis n. sp. (pag. 13.)

Kopf: Fühler etwas länger als Kopf und Thorax zusammen, die 2 Wurzelglieder gelb, das 1. und 2. Geisselglied gelblichbraun, die übrigen braun, oder auch die ganze Geissel 


\section{$2 \mathrm{BHL}$ Biodiversity Heritage Library}

Krauss, Hermann August. 1886. "Beitrag zur Kenntniss der alpinen

Orthopterenfauna." Wiener entomologische Zeitung 5, 319-326.

https://doi.org/10.5962/bhl.part.20607.

View This Item Online: https://www.biodiversitylibrary.org/item/43771

DOI: https://doi.org/10.5962/bhl.part.20607

Permalink: https://www.biodiversitylibrary.org/partpdf/20607.

\section{Holding Institution}

Smithsonian Libraries

\section{Sponsored by}

Smithsonian

\section{Copyright \& Reuse}

Copyright Status: NOT_IN_COPYRIGHT

This document was created from content at the Biodiversity Heritage Library, the world's largest open access digital library for biodiversity literature and archives. Visit BHL at https://www.biodiversitylibrary.org. 